\title{
EF-P Is Essential for Rapid Synthesis of Proteins Containing Consecutive Proline Residues
}

\author{
Lili K. Doerfel, ${ }^{1 *}$ Ingo Wohlgemuth, ${ }^{1,2 *}$ Christina Kothe, ${ }^{1}$ Frank Peske, ${ }^{1}$ Henning \\ Urlaub, ${ }^{2,3}$ Marina V. Rodnina ${ }^{1} \dagger$
}

${ }^{1}$ Department of Physical Biochemistry, Max Planck Institute for Biophysical Chemistry, 37077 Goettingen, Germany. ${ }^{2}$ Bioanalytical Mass Spectrometry Group, Max Planck Institute for Biophysical Chemistry, 37077 Goettingen, Germany. ${ }^{3}$ Bioanalytics, Department of Clinical Chemistry, University Medical Center Goettingen, 37075 Goettingen, Germany.

*These authors contributed equally to this work.

†To whom correspondence should be addressed. E-mail: rodnina@mpibpc.mpg.de

Elongation factor $P$ (EF-P) is a translation factor of unknown function which has been implicated in a great variety of cellular processes. Here we show that EF-P prevents ribosome from stalling during synthesis of proteins containing consecutive prolines, such as PPG, PPP or longer proline strings in natural and engineered model proteins. EF-P promotes peptide bond formation and stabilizes the peptidyl-tRNA in the catalytic center of the ribosome. EF-P is posttranslationally modified by a hydroxylated $\beta$-lysine attached to a lysine residue. The modification enhances the catalytic proficiency of the factor mainly by increasing its affinity to the ribosome. We propose that EF-P and its eukaryotic homolog, elF5A, are essential for the synthesis of a subset of proteins containing proline stretches in all cells.

Bacterial elongation factor P (EF-P) and its archaeal/eukaryotic homolog, initiation factor 5A (a/eIF5A), are universally conserved proteins with unknown cellular function. EF-P was reported to modulate cell viability, growth, virulence, motility, and sensitivity to low osmolarity, detergents, and antibiotics $(1,2)$. The EF-P binding site on the ribosome is located at the interface of the 30S and 50S ribosomal subunits between the binding sites for peptidyl-tRNA ( $\mathrm{P}$ site) and the exiting tRNA (E site). The N-terminal domain of EF-P interacts with the acceptor stem of the P site-bound tRNA near the peptidyl transferase center (3). Both EF$\mathrm{P}$ and eIF5A are posttranslationally modified (4-8). In Escherichia coli EF-P, lysine ${ }^{34}$ is modified by the action of three enzymes, YjeK, YjeA, and YcfM (5, 7-9). The proposed cellular function of EF-P/eIF5A is to optimize the ribosomes for more productive interactions with tRNA and release factors $(3,10-13)$. However, the magnitude of the reported effects appeared too small (less than 2-fold) for a universal function. Here we clarify the functional role of EF-P by investigating the role of the factor in the translation of various mRNA sequences.

The activity of EF-P in accelerating peptide bond formation was originally identified by its ability to increase the yield of formylmethionyl-puromycin (fMet-Pmn) synthesis $(12,13)$. As reaction rates vary by $\sim 1000$-fold for different C-terminal amino acids in the peptidyl-tRNA (14), we examined whether EF-P may specifically accelerate the product formation with poorly reactive peptidyl-tRNAs, such as those containing C-terminal proline residues (Fig. 1A and fig. S1). The formation of the tripeptide fMetPro-Pmn was accelerated by almost 90fold in the presence of EF-P, while in the other cases the reaction was stimulated by less than 5 -fold. To explore the effect of the amino acid following a proline residue, we tested Gly- and Pro-tRNAs which are known to be slow in peptide bond formation and can contribute to ribosome stalling (15-17). While EF-P enhanced peptide bond formation by less than 2fold for most combinations, large effects were observed for the formation of fMetProGly (8-fold) and fMetProPro (16-fold) (Fig. 1B). When longer strings of Pro and Gly residues were examined, such as fMPPG, fMPPGF, or fMPPPF, practically no correct product was formed in the absence of EF-P, whereas the addition of EF-P rescued the rapid production of the respective peptide (Fig. 1C). The low yield of product formation in the absence of EF-P can be explained by the loss of peptidyl-tRNA from the stalled ribosomes (e.g., fMPPtRNA; fig. S2A). These results suggested a specific effect of EF-P in accelerating the reaction with poorly reactive substrates which otherwise cause ribosome stalling.

To verify whether prolinecontaining sequences as such induce ribosome stalling that can be rescued by EF-P, we engineered PG, PP, PPG, and $\mathrm{PPP}$ sequences into an N-terminal fragment of protein PrmC, which originally does not contain such sequences and is rapidly synthesized independent of the presence of EF-P (Fig. 2). Introducing a glycine residue following Pro $^{20}$ resulted in the ribosome pausing, as shown by the accumulation of a peptide of about 20 amino acids; however pausing was transient and not affected by EF-P. When a second proline residue preceding Pro $^{20}$ was engineered, the ribosome paused at the ProPro sequence, and EF-P reduced the pause time from 20 to $10 \mathrm{~s}$. When PPG or PPP sequences were introduced, the ribosomes were stalled and essentially no full-length product was produced. The ribosomes stalled upon translation of the PPG sequence contained a ProPro-ending peptidyltRNA in the P site and a Gly-tRNA in the A site (fig. S2B). Addition of EF-P rescued the synthesis of full-length product by preventing or alleviating ribosome stalling (Fig. 2 and fig. S2C).

The sequences PPG and PPP as well as longer proline stretches are found in a large number of cellular proteins, raising the question of whether they require EF-P for their synthesis. We tested the effect of EF$\mathrm{P}$ on the synthesis of some of these proteins, such as TonB, YafD, Rz1, and AmiB (Fig. 3). In the absence of EF-P, translation was stalled at the consecutive prolines, and the addition of EF-P alleviated ribosome stalling, resulting in the rapid synthesis of the respective full-length peptide. When the Pro stretches were particularly long, as in AmiB or Rz1, very little full-length product was synthesized in the absence of EF-P, as translation was halted at the stalling site. In all cases, the addition of EF$P$ resulted in the efficient synthesis of full-length protein. Transient pauses that occurred at sequences other than PPP/PPG were not alleviated by EF-P (Fig. 3).

Posttranslational modification of EF-P appears crucial for the factor's function $(9,18)$. Translation of the fMPPG sequence, which was abolished in the absence of EF-P, was fully restored with EF-P carrying the $\beta$-lysine modification at Lys ${ }^{34}$; further hydroxylation did not alter the activity, whereas the absence of modifications reduced the activity of the 
factor (Fig. 4). A similar effect was observed for the translation of the PPG sequence in PrmC (fig. S4). Analysis of the reaction kinetics indicated that the modification increased the affinity of EF-P binding to the ribosome 30 -fold (Fig. 4). The maximum rate of fMPPG synthesis decreased $\sim 4$-fold with unmodified, compared to modified EF-P, resulting in a $>100$-fold difference in $k_{\text {cat }} / K_{\mathrm{M}}$. The low catalytic proficiency of unmodified EF-P may explain why deletions of the yjeA or yjeK genes that code for the modification enzymes lead to phenotypes that are similar to, or only somewhat milder than, the deletion of efp, the gene coding for EF-P $(1,2)$. On the other hand, even in the absence of active EF-P, small amounts of proteins with proline stretches are slowly formed (Fig. 3 ); this may be sufficient to support the viability of strains in which the efp, yieA, and yieK genes are deleted.

Our data indicate that EF-P is a translation factor that promotes the synthesis of proteins containing PPG, PPP, and longer polyproline stretches by preventing ribosome stalling during the formation of proline-proline or proline-glycine peptide bonds. Thus, EF-P (and likely eIF5A) can be considered a third universal translation elongation factor that acts on the ribosome, in addition to the two factors, EF-Tu and EF$\mathrm{G}$, which catalyze the mRNA decoding and tRNA translocation steps, respectively. In contrast to the latter two factors, which act in each elongation cycle irrespective of the amino acid incorporated, EF-P/eIF5A are responsible for the elongation of a subset of nascent proteins and are the only factors identified so far that augment the peptidyl transferase activity of the ribosome.

The pyrrolidine ring of proline restricts the number of accessible conformations for this residue and may impose structural constraints on the positioning of the amino acid in the peptidyl transferase center, resulting in slow peptide bond formation and, thereby, ribosome stalling. EF-P facilitates peptide bond formation by stabilizing peptidyl-tRNA on the ribosome and possibly by promoting optimal positioning of the substrates. The cellular fate of the nascent peptide on stalled ribosomes is likely to depend on its length: shorter peptides may be lost due to peptidyl-tRNA drop-off from the ribosome $(16,19)$, whereas ribosomes stalled after the synthesis of longer peptides might be rescued through SsrA-, ArfA- or YaeJ-dependent pathways (20-23).

Of more than 4000 annotated E. coli proteins, about 270 contain motifs of three or more consecutive prolines or PPG motifs (table S1). Among those, proteins belonging to the basal transcription-translation machinery are underrepresented, whereas metabolic enzymes, transporters, and regulatory transcription factors are frequent. The observed pleiotropic effects of efp, yjeA or yjeK deletions (1) can be rationalized by the impaired synthesis of proteins with proline stretches. For example, decreased synthesis of TonB, a protein from the EF-P interactome (24) which supplies energy for the function of TonB-dependent transporters, may result in a cumulative inhibition of transport processes (25). The severe effects of efp, yjeA or yjeK deletions on cell motility may be explained by the failure to support the coordinated expression of flagellar proteins FlhC, FliF, and Flk, all containing PPP and PPG motifs, whereas the deficiency of EF-P mutants in utilizing $\gamma$-glutamyl-glycine as a nitrogen source (1) may be caused by impaired synthesis of the corresponding catabolic enzyme, $\gamma$-glutamyl-transpeptidase, which contains a PPP motif. Finally, EF-P may affect the translation of virulence proteins, such as protein EspF, which is a key player during the infection of eukaryotic hosts by enterohaemorrhagic and enteropathogenic strains of $E$. coli (EHEC and EPEC) (26, 27). EspF contains several proline runs, including PPPP sequences, in its functionally important SH3 binding domains (27). Given that the posttranslational modifications of eukaryotic eIF5A and bacterial EF-P are different, the occurrence of proline runs in many pathogenic proteins makes EF-P and its modification enzymes promising new targets for developing highly specific, potent antimicrobials.

\section{References and Notes}

1. S. B. Zou et al., Loss of elongation factor $\mathrm{P}$ disrupts bacterial outer membrane integrity. J. Bacteriol. 194, 413 (2012). doi:10.1128/JB.05864-11 Medline

2. S. B. Zou, H. Roy, M. Ibba, W. W. Navarre, Elongation factor P mediates a novel post-transcriptional regulatory pathway critical for bacterial virulence. Virulence 2, 147 (2011). doi:10.4161/viru.2.2.15039 Medline

3. G. Blaha, R. E. Stanley, T. A. Steitz, Formation of the first peptide bond: the structure of EF-P bound to the 70S ribosome. Science 325, 966 (2009). doi:10.1126/science.1175800 Medline

4. E. C. Wolff, K. R. Kang, Y. S. Kim, M. H. Park, Posttranslational synthesis of hypusine: Evolutionary progression and specificity of the hypusine modification. Amino Acids 33, 341 (2007). doi:10.1007/s00726-007-0525-0 Medline

5. T. Yanagisawa, T. Sumida, R. Ishii, C. Takemoto, S. Yokoyama, A paralog of lysyl-tRNA synthetase aminoacylates a conserved lysine residue in translation elongation factor P. Nat. Struct. Mol. Biol. 17, 1136 (2010). doi:10.1038/nsmb.1889 Medline

6. M. Bailly, V. de Crécy-Lagard, Predicting the pathway involved in posttranslational modification of elongation factor $\mathrm{P}$ in a subset of bacterial species. Biol. Direct 5, 3 (2010). doi:10.1186/1745-6150-5-3 Medline

7. H. Roy et al., The tRNA synthetase paralog PoxA modifies elongation factor-P with (R)- $\beta$-lysine. Nat. Chem. Biol. 7, 667 (2011). doi:10.1038/nchembio.632 Medline

8. L. Peil et al., Lys34 of translation elongation factor EF-P is hydroxylated by YfcM. Nat. Chem. Biol. 8, 695 (2012). doi:10.1038/nchembio.1001 Medline

9. W. W. Navarre et al., PoxA, yjeK, and elongation factor P coordinately modulate virulence and drug resistance in Salmonella enterica. Mol. Cell 39 209 (2010). doi:10.1016/i.molcel.2010.06.021 Medline

10. B. R. Glick, S. Chládek, M. C. Ganoza, Peptide bond formation stimulated by protein synthesis factor EF-P depends on the aminoacyl moiety of the acceptor. Eur. J. Biochem. 97, 23 (1979). doi:10.1111/j.14321033.1979.tb13081.x Medline

11. B. R. Glick, M. C. Ganoza, Identification of a soluble protein that stimulates peptide bond synthesis. Proc. Natl. Acad. Sci. U.S.A. 72, 4257 (1975) doi:10.1073/pnas.72.11.4257 Medline

12. P. Saini, D. E. Eyler, R. Green, T. E. Dever, Hypusine-containing protein eIF5A promotes translation elongation. Nature 459, 118 (2009) doi:10.1038/nature08034 Medline

13. A. P. Gregio, V. P. Cano, J. S. Avaca, S. R. Valentini, C. F. Zanelli, eIF5A has a function in the elongation step of translation in yeast. Biochem. Biophys. Res. Commun. 380, 785 (2009). doi:10.1016/j.bbrc.2009.01.148 Medline

14. I. Wohlgemuth, S. Brenner, M. Beringer, M. V. Rodnina, Modulation of the rate of peptidyl transfer on the ribosome by the nature of substrates. J. Biol. Chem. 283, 32229 (2008). doi:10.1074/jbc.M805316200 Medline

15. M. Johansson et al., pH-sensitivity of the ribosomal peptidyl transfer reaction dependent on the identity of the A-site aminoacyl-tRNA. Proc. Natl. Acad. Sci. U.S.A. 108, 79 (2011). doi:10.1073/pnas.1012612107 Medline

16. M. Y. Pavlov et al., Slow peptide bond formation by proline and other Nalkylamino acids in translation. Proc. Natl. Acad. Sci. U.S.A. 106, 50 (2009). doi:10.1073/pnas.0809211106 Medline

17. D. R. Tanner, D. A. Cariello, C. J. Woolstenhulme, M. A. Broadbent, A. R. Buskirk, Genetic identification of nascent peptides that induce ribosome stalling. J. Biol. Chem. 284, 34809 (2009). doi:10.1074/jbc.M109.039040 Medline

18. J. H. Park et al., Post-translational modification by $\beta$-lysylation is required for activity of Escherichia coli elongation factor P (EF-P). J. Biol. Chem. 287, 2579 (2012). doi:10.1074/jbc.M111.309633 Medline

19. V. Heurgué-Hamard et al., Ribosome release factor RF4 and termination factor RF3 are involved in dissociation of peptidyl-tRNA from the ribosome. EMBO J. 17, 808 (1998). doi:10.1093/emboj/17.3.808 Medline

20. Y. Chadani, K. Ono, K. Kutsukake, T. Abo, Escherichia coli YaeJ protein mediates a novel ribosome-rescue pathway distinct from SsrA- and ArfAmediated pathways. Mol. Microbiol. 80, 772 (2011). doi:10.1111/j.13652958.2011.07607.x Medline

21. Y. Chadani et al., Ribosome rescue by Escherichia coli ArfA (YhdL) in the absence of trans-translation system. Mol. Microbiol. 78, 796 (2010) doi:10.1111/j.1365-2958.2010.07375.x Medline

22. C. S. Hayes, B. Bose, R. T. Sauer, Proline residues at the $\mathrm{C}$ terminus of nascent chains induce SsrA tagging during translation termination. J. Biol. 
Chem. 277, 33825 (2002). doi:10.1074/jbc.M205405200 Medline

23. T. Sunohara, T. Abo, T. Inada, H. Aiba, The C-terminal amino acid sequence of nascent peptide is a major determinant of SsrA tagging at all three stop codons. RNA 8, 1416 (2002). doi:10.1017/S1355838202020198 Medline

24. L. Salwinski et al., The Database of Interacting Proteins: 2004 update. Nucleic Acids Res. 32, D449 (2004). doi:10.1093/nar/gkh086 Medline

25. N. Noinaj, M. Guillier, T. J. Barnard, S. K. Buchanan, TonB-dependent transporters: Regulation, structure, and function. Annu. Rev. Microbiol. 64, 43 (2010). doi:10.1146/annurev.micro.112408.134247 Medline

26. N. A. Sallee et al., The pathogen protein $\operatorname{EspF(U)~hijacks~actin~polymerization~}$ using mimicry and multivalency. Nature 454, 1005 (2008). doi:10.1038/nature07170 Medline

27. A. Holmes, S. Mühlen, A. J. Roe, P. Dean, The EspF effector, a bacterial pathogen's Swiss army knife. Infect. Immun. 78, 4445 (2010). doi:10.1128/IAI.00635-10 Medline

28. H. Aoki, S. L. Adams, M. A. Turner, M. C. Ganoza, Molecular characterization of the prokaryotic efp gene product involved in a peptidyltransferase reaction. Biochimie 79, 7 (1997). doi:10.1016/S03009084(97)87619-5 Medline

29. M. V. Rodnina, W. Wintermeyer, GTP consumption of elongation factor Tu during translation of heteropolymeric mRNAs. Proc. Natl. Acad. Sci. U.S.A. 92, 1945 (1995). doi:10.1073/pnas.92.6.1945 Medline

30. C. Schmidt, C. Lenz, M. Grote, R. Lührmann, H. Urlaub, Determination of protein stoichiometry within protein complexes using absolute quantification and multiple reaction monitoring. Anal. Chem. 82, 2784 (2010). doi:10.1021/ac902710k Medline

31. K. B. Gromadski, M. V. Rodnina, Kinetic determinants of high-fidelity tRNA discrimination on the ribosome. Mol. Cell 13, 191 (2004). doi:10.1016/S10972765(04)00005-X Medline

32. U. Kothe, A. Paleskava, A. L. Konevega, M. V. Rodnina, Single-step purification of specific tRNAs by hydrophobic tagging. Anal. Biochem. 356, 148 (2006). doi:10.1016/j.ab.2006.04.038 Medline

33. I. Wohlgemuth, C. Pohl, M. V. Rodnina, Optimization of speed and accuracy of decoding in translation. EMBO J. 29, 3701 (2010). doi:10.1038/emboj.2010.229 Medline

Acknowledgments: We thank $\mathrm{W}$. Wintermeyer for critically reading the manuscript, W. Holtkamp and J. Mittelstaet for the translation assay and M1 and M2 peptide markers, D. Görlich and C. Enke for the antibody against EFP, and O. Geintzer, S. Kappler, T. Wiles, M. Zimmermann, T. Uhlendorf, A. Bursy, and U. Plessmann for expert technical assistance. The work was funded by the Max Planck Society and by grants of the Deutsche Forschungsgemeinschaft.

\section{Supplementary Materials}

www.sciencemag.org/cgi/content/full/science.1229017/DC1

Materials and Methods

Figs. S1 to S4

Table S1

References (28-33)

20 August 2012; accepted 26 October 2012

Published online 13 December 2012

10.1126/science.1229017 

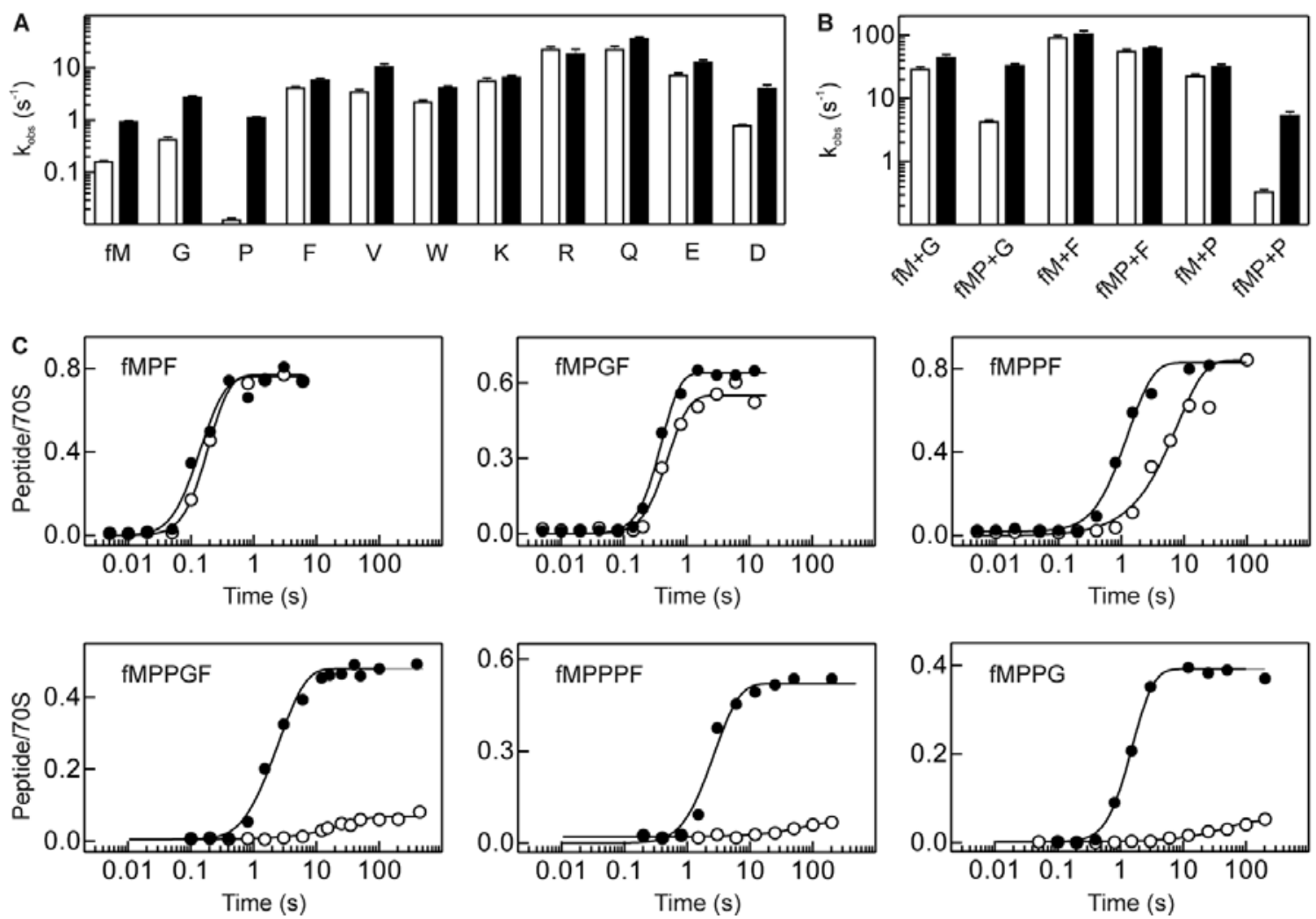

Fig. 1. EF-P promotes the synthesis of Pro and Gly-containing peptides on the ribosome. (A) Rates of peptide bond formation between fMet-tRNA ${ }^{\text {fMet }}$ or fMetX-tRNA ${ }^{X}$ and Pmn, where $X$ stands for different amino acids, as indicated, in the absence (white bars) or presence (black bars) of EF-P $(3 \mu \mathrm{M})$. (B) Rates of peptide bond formation between fMet-tRNA (fM) or fMetPro-tRNA (fMP) in the $P$ site and Gly-tRNA ${ }^{\text {Gly }}$, Phe-tRNA ${ }^{\text {Phe }}$, or Pro-tRNA ${ }^{\text {Pro }}$ (G, F, and P, respectively) in the A site. (C) Formation of model oligopeptides in a reconstituted translation system in the absence (open circles) and presence (closed circles) of EF-P. 


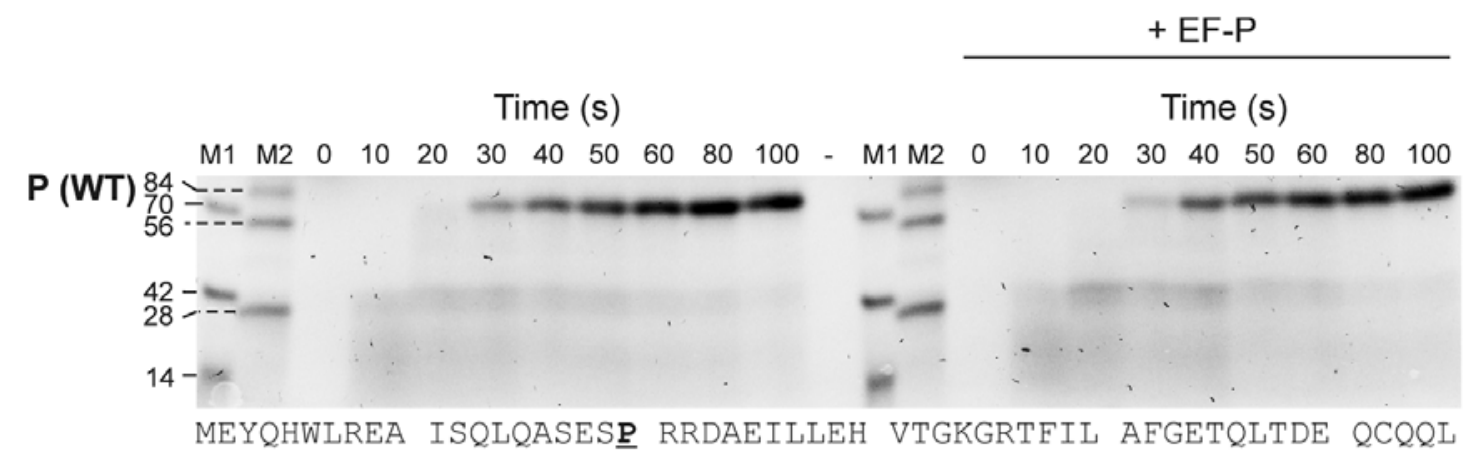

PG

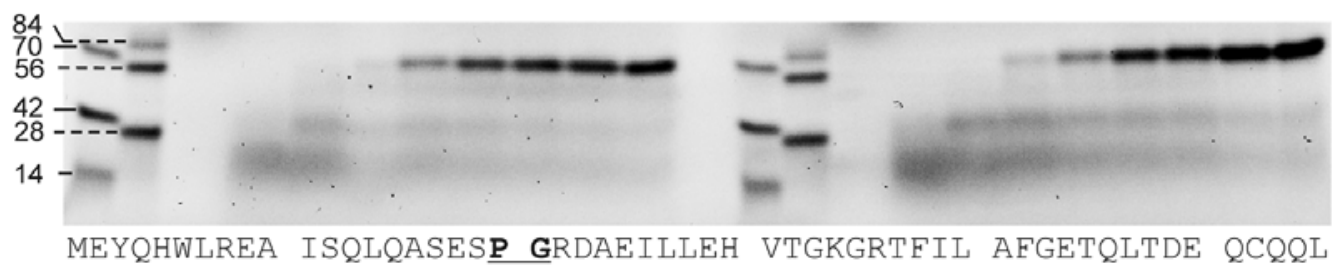

PP

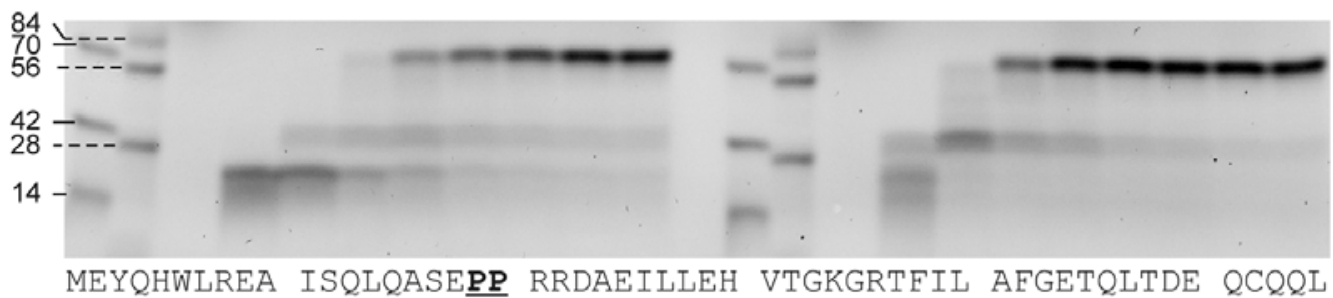

PPG

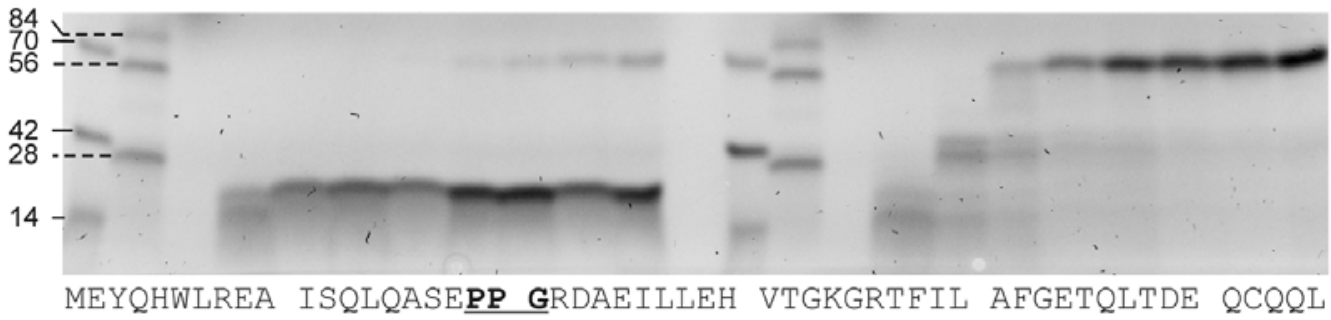

PPP

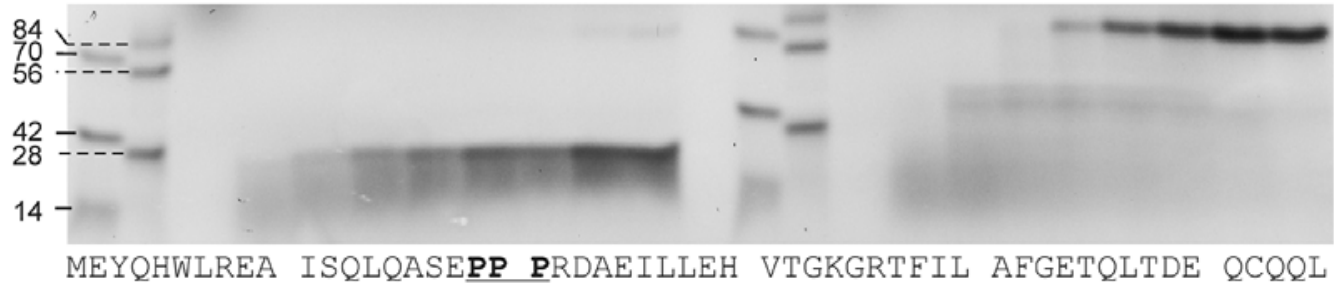

Fig. 2. EF-P prevents ribosome stalling on PPG and PPP sequences engineered into PrmC. Translation of the N-terminal domain of PrmC (75 amino acids) with wild-type (wt) or mutant sequences containing PG, PP, PPG or PPP in the absence or presence of EF-P. Peptides were separated by SDS-PAGE and visualized by the fluorescence of BODIPY-FL attached to the $\mathrm{N}$ terminus of the peptides. M1 and M2 are peptide markers for PrmC fragments of the indicated number of amino acids. 

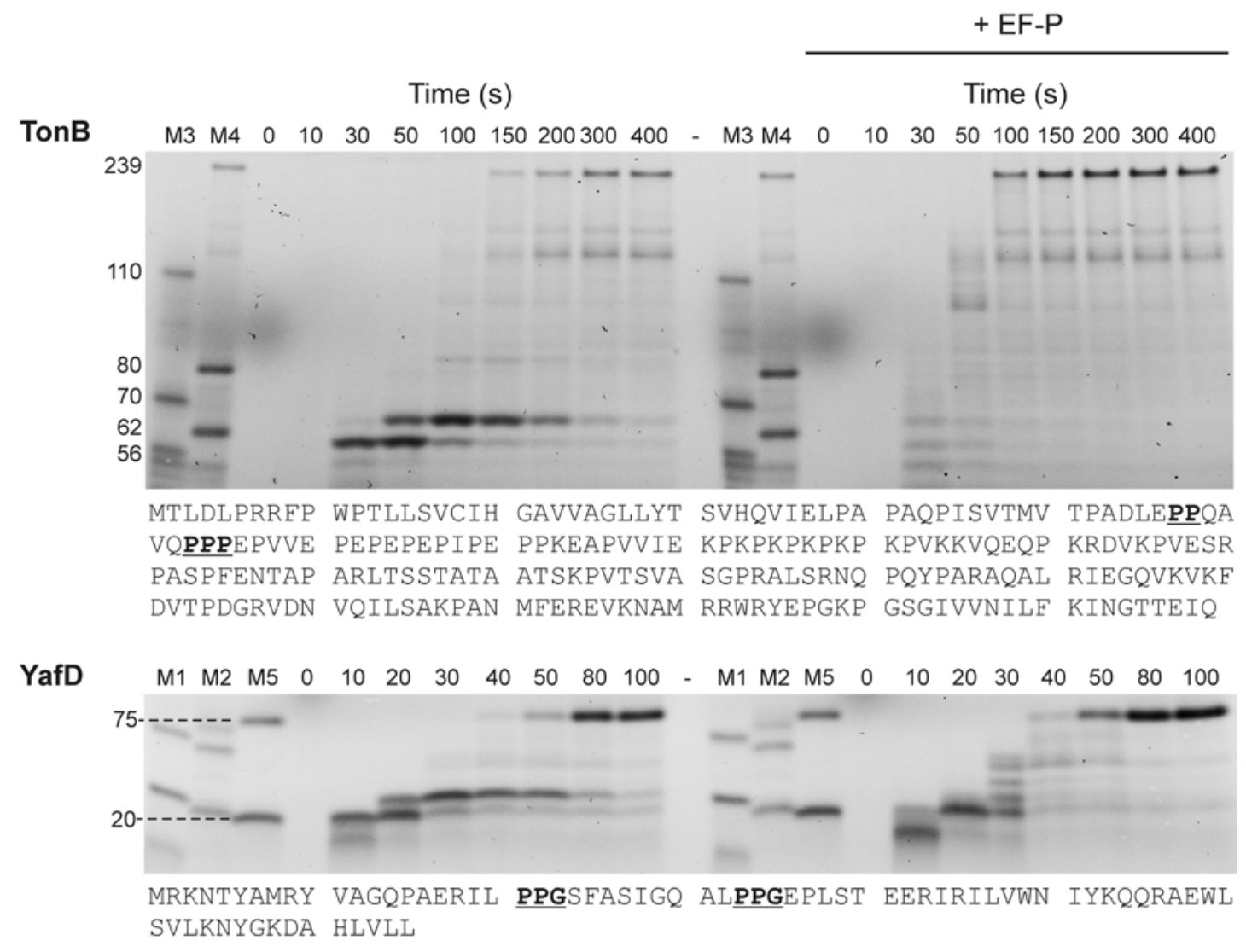

$\begin{array}{lllllllllllllllllllllll}R z 1 & \text { M1 M2 M6 } & 0 & 10 & 20 & 30 & 40 & 50 & 60 & 80 & - & \text { M1 M2 M6 } & 0 & 10 & 20 & 30 & 40 & 50 & 60 & 80\end{array}$

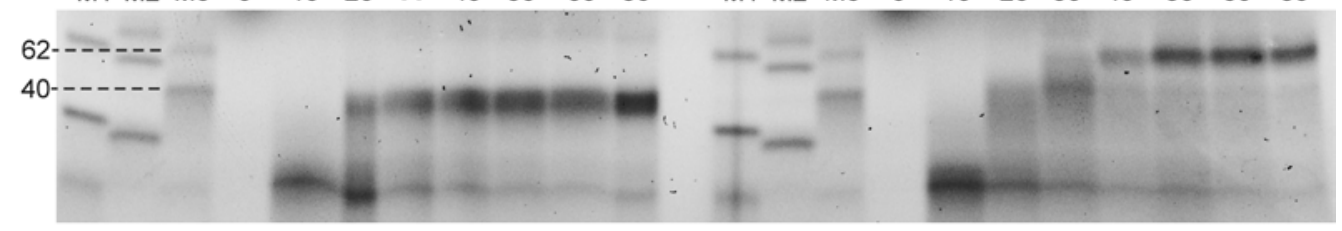

MRKLKMMLCV MMLPLVVVGC TSKQSVSQCV KPPPPPAWIM QPPPDWQTPL NGIISPSGND W

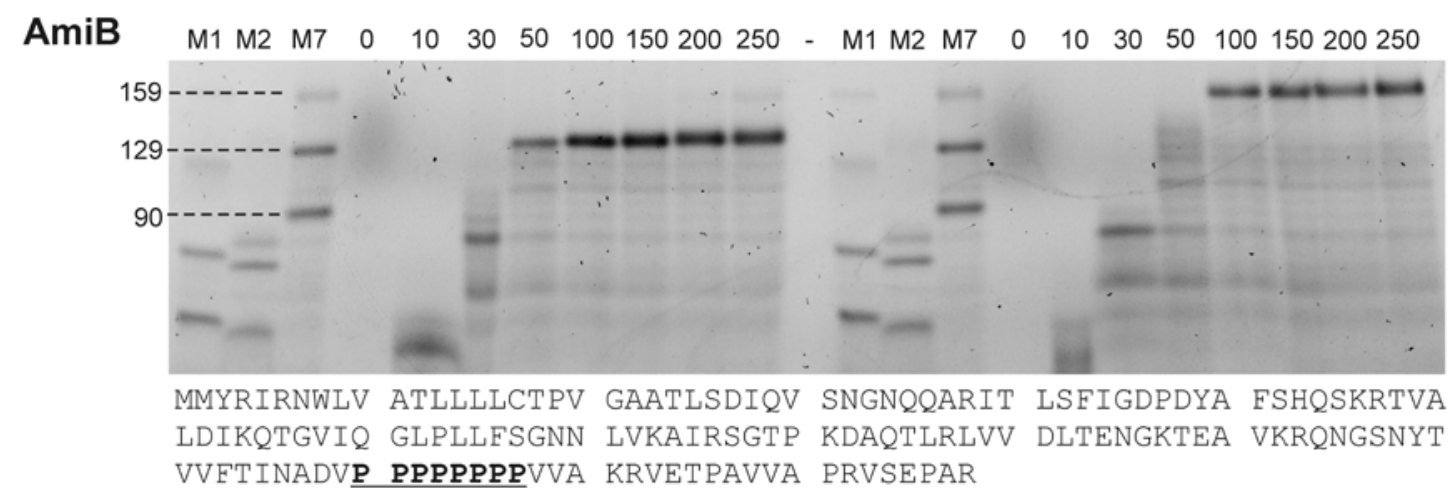

Fig. 3. EF-P alleviates PPP/PPG-induced stalling during synthesis of natural proteins. Translation products of TonB (239 amino acids), YafD (75 amino acids from the $\mathrm{N}$ terminus), Rz1 (62 amino acids), and AmiB (159 amino acids from the $\mathrm{N}$ terminus) were separated by SDS-PAGE. M3, and M4, peptide markers containing TonB fragments of the indicated lengths. M5, M6, and M7, peptide markers of the indicated lengths of YafD, RZ1, and AmiB sequences, respectively. 

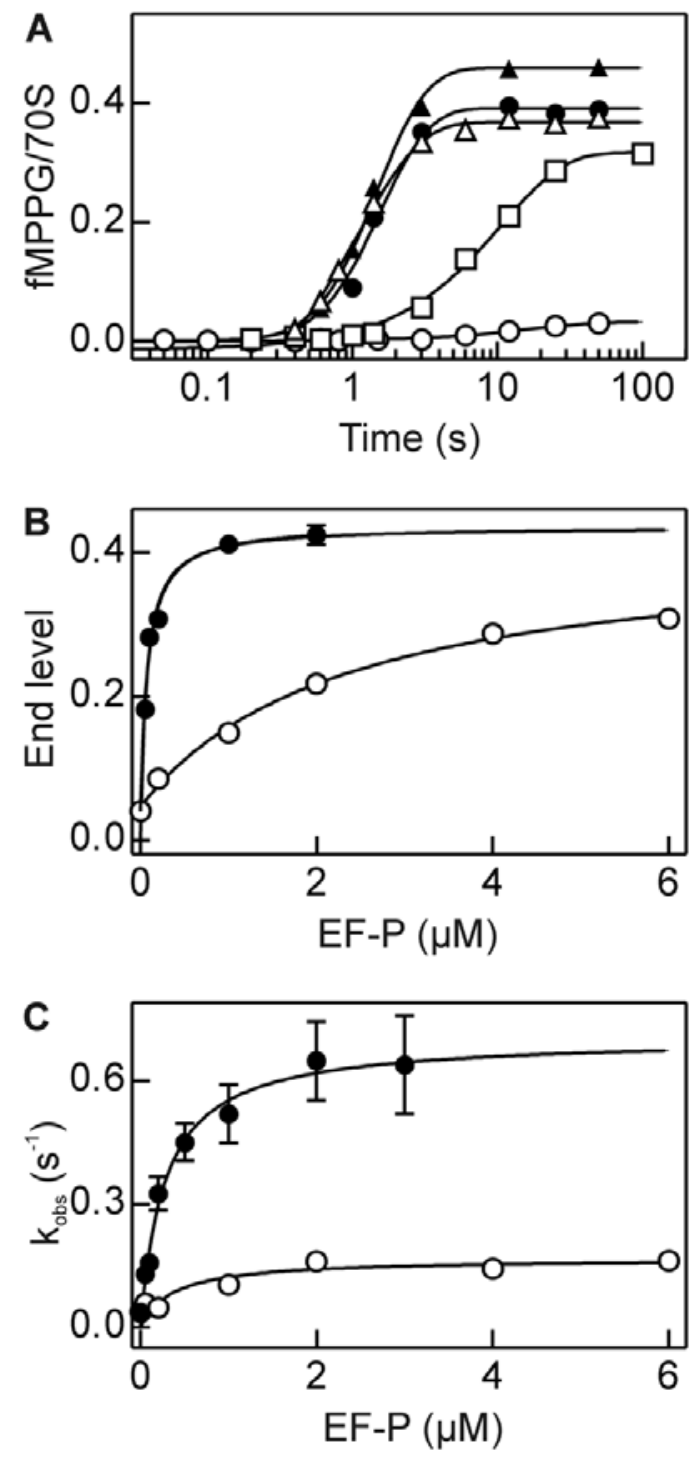

Fig. 4. Contribution of Lys ${ }^{34}$ modifications to EF-P activity. (A) Time courses of fMPPG synthesis. The formation of the tetrapeptide was measured in the absence of EF-P (open circles), in the presence of unmodified EF-P (open squares), overexpressed lysinylated but unhydroxylated EF-P (closed triangles), and lysinylated/hydroxylated overexpressed EF-P (closed circles) or native EF-P (open triangles). The extent of the respective modification was verified by mass spectrometry (fig. S3). (B) Yield of fMPPG peptide as a function of EF$\mathrm{P}$ concentration with unmodified (open circles; $K_{1 / 2}=2.4 \pm 0.5 \mu \mathrm{M}$ ) or fully modified (closed circles; $K_{1 / 2}=0.08 \pm 0.02 \mu \mathrm{M}$ ) EF-P. $K_{1 / 2}$ is the EF-P concentration at which $50 \%$ of the maximum yield is reached. (C) EF-P concentration dependence of $\mathrm{AMPPG}$ synthesis rate with unmodified EF-P (open circles; $k_{\text {cat }}=0.12 \pm 0.03 \mathrm{~s}^{-1}$ ) and fully modified EF-P (closed circles; $k_{\text {cat }}=0.65 \pm 0.02 \mathrm{~s}^{-1}$ ); $k_{\text {cat }}$ is the maximum reaction rate obtained by hyperbolic fitting. 